Historic, Archive Document

Do not assume content reflects current scientific knowledge, policies, or practices. 

THE BELT SEED COMPANY

IN CORPORATED

BALTIMORE, MD.

RETURN POSTAC

GUARANTEE:

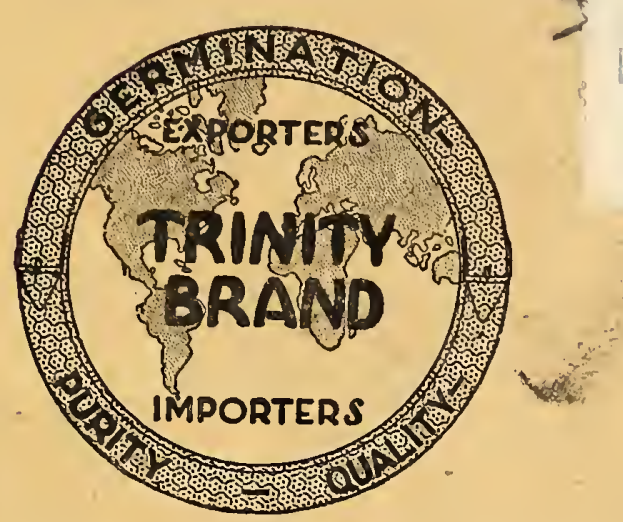

जBC. $4351 / 2$ P. L. 63 R.

U. S. Postagr PAID

Baltimore, MD.

Permit No. 378

U. S. Department of AGriculture,

D. N. Shoemaker

WASIIINGTON, D. C.

BUREAU OF PLANT INDUSTRY, (Hott

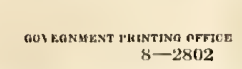

Fin $\mathrm{F} Y$ NGAR 1 in:

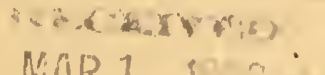

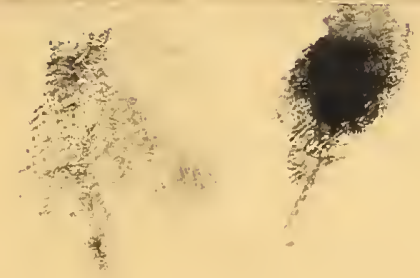

$\sqrt{4}$ 


\section{GEOGRAPHY NOTES}

The red clover seed producing area in Poland lies along the backbone of the Continental European divide. The elevation is high and the winters very severe. Average winter temperature is 5 degrees below zero. It lies nearer the Arctic Circle than any point in the United States, no matter how fal' north. Poland has supplied the bulk of the adapted clover imported into the United States the last two years.

M. T. Munn, New York State Seed Analyst, says there is no such thing as a bargain in seeds.

\section{FROM-THE ARCHIVES}

In 1919-20, the United States used 20 million pounds of imported clover plus a normal domestic crop at an average price level of 50-60 cents per pound. Will this year's price level of 17-23 cents encourage consumption of supplies that are probably no larger?

\section{ALFALFA 3 FT. HIGH}

Monticello, Fla. Although outside what is normally considered the Temperate Zone forage belt, we are growing here Alfalfa three ft. high, Sweet five and splendid crops of Red, White and Alsyke.

\section{U.S. DEPT. AGRICULTURE OUTLOOK SOLVES CLOVER PROBLEM}

U.S. D. A. outlook report on clovers and alfalfa suggests every producing and consuming farmer holding or buying two years supply of seed. If this is good medicine for the farmer, it ought to be for the seed dea er.

U. S. D. A. says cowpea crop is half sold with prices approximately $25 \%$ higher than the five-year average.

Soybeans, too, are half sold, but prices vary little above the five-year average.

\section{SOCRATES SAYS}

The Agricultural Outlook of 1930 as issued by the United States Department of Agriculture indicates only average prices and prospects for 1930 unless production is kept down. There is only one answer. Reduce the cost of what is produced by maintaining fertility, thus increasing acre yields. Better seeds (i. e. Trinity Brand) are the foundation of any cost reduction program.

\section{BABSON AGAIN}

Roger Babson says the farming sections offer the best sales opportunities at the present time.

We quote today's values, wholesale quantities, f.o.b. Baltimore, subject market changes and prior sale. Bags not returnable. Terms: NET CASH.

\section{RED CLOVER}

\section{ADAPTED*}

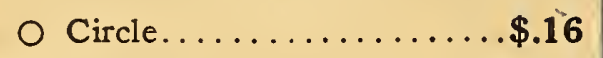

$\square$ Square

$\nabla$ Trinity.

$\nabla$ Trinity 99.60 .

\section{DOMESTIC}

SAPLING or MAMMOTH*

$\nabla$ Trinity 99.60 .

\section{ALSYKE*}

\section{Circle $96 \%$}

$\nabla$ Trinity $99 \%$

\section{TIMOTHY*}

- Circle $98 \%$ $\$ 3.15$

$\square$ Square $99 \%$.

$\nabla$ Trinity $99.60 \%$

3.35

\section{U. S. VERIFIED ALFALFA*} DODDERFREE

$\nabla$ Kansas 99.60 .

$\nabla$ Northwestern 99.5

$\nabla$ N.W. Grimm .

$\nabla$ WHITE CLOVER 97\%* . . 24

O SWEET CLOVER*

$\nabla$ SWEET CLOVER $99 \frac{1}{2} *$

$\nabla$ GRUNDY CO. SWEET*.

$\nabla$ JAPAN CLOVER**

.08

.17

\section{KENTUCKY BLUEGRASS**}

$\square$ Square 19\#f 78/78 ..... \$.20

$\nabla$ Trinity $21 \# 82 / 82 \ldots \ldots \ldots .21$

$\nabla$ Canada............... 18

\section{REDTOP-HERDS GRASS*}

$\square$ Square $90 \% \ldots \ldots \ldots \ldots \ldots .16 \frac{1}{2}$

$\nabla$ Trinity $95 \% \ldots \ldots \ldots \ldots . .17$

$\nabla$ Unhulled $40 \% \ldots \ldots \ldots . . .09$

FARM and TURF GRASSES**

$\square$ Pasture Mixture..........12

$\nabla$ Pasture Mixture.......... .20

Tall Oat Grass............ 16

Bermuda Grass.......... .35

Carpet Grass.......... .20

DOMESTIC RYEGRASS. . .07

Imported Italian Ryegrass. . .10

Imported English Ryegrass. .10

Imported Pacey's Ryegrass. .12

Poa Trivialis..........38

Chewings Fescue.........28

Sheep Fescue. . . . . . . . . . .22

Red Fescue............. .25

$\nabla$ German Bentgrass 90/90... .60

$\nabla$ Colonial Bentgrass 98/95 . . 60

$.07 \frac{1}{2} \square$ Square domestic..........16 $\nabla$ Danish onion free $92 / 95 \ldots . .19$

$\nabla$ SUDAN GRASS**......06 $\frac{1}{2}$

\section{LAWN GRASSES** \\ Trinity 100\# bags......\$.28 \\ Trinity $100 \#$ in $5 \#$ bags.... . 30 \\ Evergreen 100\# bags...... .20 \\ Evergreen 100\# in 3\# bags. . . .22 \\ Velvet Lawngrass $100 \#$ bags .16 \\ Shady Lawngrass $100 \#$ bags .30 \\ $\nabla$ WHITE CLOVER....... 24 \\ SHEEP MANURE . . . . 2.00 \\ BONEMEAL . . . . . . . 2.25 \\ Crab Grass Cure. . . . . . 1.00 \\ Vigoro............... 3.50 \\ Peat Moss per bale. ...... 2.00}

\section{E. RAPE**}

Holland Grown.......\$.07

Japanese Grown . . . . . . . . .06

$\nabla$ NEW CRIMSON.........

$\nabla$ HAIRY VETCH imported.

$\nabla$ HAIRY VETCH domestic.

$\nabla$ AUSTRIAN PEAS. . . . . .

SEED GRAIN***

Eureka Ensilag Corn.....2.50

Lancaster Surecrop Corn. 2.25

Reids Yellow Dent Corn...2.25

Boone Co.White Corn . . . . 2.25

Sweepstakes Corn.......2.50

Spring Rye..........2.00

Spring Wheat..........2.25

Spring Barley . . . . . . . . 1.50

Heavy Oats........... .80

PACKING $\left\{\begin{array}{l}* \text { Cotton Bags }-35 \mathrm{c}-\text { net weight } \\ * * \text { Burlap }\end{array}\right.$ ** Burlap Sax-free-gross weight 